\title{
ELECTRONIC SMOG IN EUROPE
}

\author{
H.C. KAHLMANN
}

Netherlands Foundation for Radio Astronomy

Dwingeloo, The Netherlands

\begin{abstract}
With so many satellites (current and anticipated) broadcasting all sorts of signals in all sorts of bands, a group of radio astronomers came together in 1987 to discuss the problems. This group has become the European Science Foundation Committee on Radio Astronomical Frequencies (ESF-CRAF). The terms of reference of this Committee, together with its future activities and possibilities within the European legal context were discussed in the oral paper.
\end{abstract}

